\title{
DISORDER INDUCED BCS-BEC CROSSOVER
}

\author{
AYAN KHAN \\ Research Center for Dielectric and Advanced Matter Physics, \\ Pusan National University, Busan, 609-735, South Korea \\ ayan.khan@pusan.ac.kr
}

\begin{abstract}
Of late, the study of BCS-BEC crossover in the presence of weak random impurity is an interesting issue. In this proceedings we study the effect of this disorder which is included through the Nozières and Smith-Rink theory of superconducting fluctuations. In the weak regime, the random potential leaves an effect on the superconducting order parameter but it spares the chemical potential. Here we present the exact behavior of the mean field quantities as a function of the disorder by self-consistently solving the coupled equations.
\end{abstract}

Keywords: BCS-BEC Crossover; Disorder.

PACS numbers: 05.30.Jp, 74.20.Fg, 74.40.+k

\section{Introduction}

The smooth evolution of atoms from Cooper pairs to the composit bosons offers an exciting domain of research in current days. The exhilarating transformation of ultracold atomic gases can easily be viewed by changing inter-atomic low energy scattering length by means of Fano-Feshbach resonance ${ }^{1}$. This enabled us to study the Bardeen-Cooper-Schrieffer-Bose-Einstein-Condensate(BCS-BEC) crossover more closely ${ }^{2,3}$.

The recent experimental developments widened the domain for more intriguing aspects in ultracold atomic gases, the effect of impurity driven disorder is among them. In the seminal work of Anderson ${ }^{4}$, the localization effect due to impurity was predicted for the superconductors when impurity concentration is pretty high but this feature spares the material when it is less dirty. But a direct observation of Anderson localization in electronic systems is very difficult. On the contrary, ultracold atoms allow one to address the core of the phenomenon that Anderson had discovered, since they are genuine quantum particles described as matter waves, and interactions can be reduced at a negligible level so that one can study single particle behaviors. More over one can create disorder with optics and this lends a great amount of controllability and tunability in the system. Recently ultracold Bose gas in one dimension $\left({ }^{87} R b\right.$ and $\left.{ }^{39} K\right)$ enabled us to see the localization directly ${ }^{5,6}$. Latest experiments are conducted in three dimension for both non interacting atomic Fermi 
gas of ${ }^{40} K^{7}$ and Bose gas of ${ }^{87} R b^{8}$. These experiments have widened the possibility to study the crossover in the light of disorder ${ }^{9}$ experimentally.

In theoretical front, the static disorder in Fermi and in Bose systems are not a very new topic. A considerable amount of attention has been paid to disorder driven superconductors ${ }^{10,11,12}$ and in Bose gas ${ }^{13,14}$. More detail references on disordered superconductors and Bose gas can be found in Ref.[9]. Of late the interest at unitarity is also gaining pace ${ }^{15,16,17,18}$, but still it lacks the required amount of attention. Here, at present our investigation is on BCS-BEC crossover with weak uncorrelated disorder at zero temperature. To absorb the impurity in the mean field approach we follow Nozières and Smith-Rink (NSR) theory ${ }^{19}$ of superconducting fluctuations. As a result we are able to construct the modified gap and density equation $^{15}$. This enables us to solve the coupled equation self consistently as a function of the random external potential through out the crossover. At zero temperature which is not yet reported according to best of our knowledge.

We arrange our study in the following way, in Section 2 we present the formalism to include fluctuation in the mean field level. As mentioned above, very few article are available in this field at present therefore we prefer to spend some time here to discuss the intricacies. Section 3 is dedicated to our results and we draw our conclusion in Section 4.

\section{Formalism}

To describe the effect of impurity in Fermi superfluid from BCS to BEC regime one need to start from the real space Hamiltonian in three dimension for s-wave superfluid,

$$
\begin{aligned}
\mathcal{H}(\mathbf{x})= & \sum_{\sigma} \Psi_{\sigma}^{\dagger}(\mathbf{x})\left[-\frac{\nabla^{2}}{2 m}-\mu+V_{d}(\mathbf{x})\right] \Psi_{\sigma}(\mathbf{x}) \\
& +\int d x^{\prime} V\left(\mathbf{x}, \mathbf{x}^{\prime}\right) \Psi_{\uparrow}^{\dagger}\left(\mathbf{x}^{\prime}\right) \Psi_{\downarrow}^{\dagger}(\mathbf{x}) \Psi_{\downarrow}(\mathbf{x}) \Psi_{\uparrow}\left(\mathbf{x}^{\prime}\right),
\end{aligned}
$$

where $\Psi_{\sigma}^{\dagger}(\mathbf{x})$ and $\Psi_{\sigma}(\mathbf{x})$ represents the creation and annihilation of fermions with mass $m$ and spin state $\sigma$ at $\mathbf{x}$ respectively, $V_{d}(\mathbf{x})$ signifies the random potential and $\mu$ is the chemical potential. All physical constants are are taken care using Plank units. In the interaction Hamiltonian the s-wave fermionic interaction is defined with $V\left(\mathbf{x}, \mathbf{x}^{\prime}\right)=-g \delta\left(\mathbf{x}-\mathbf{x}^{\prime}\right)$ and $g$ is the bare coupling strength of fermion-fermion pairing. We choose the disorder as uncorrelated which reflects as white-noise correlations. By definition $V_{d}(\mathbf{x})=\sum_{i} g_{d} \delta\left(\mathbf{x}-\mathbf{x}_{i}\right)$ where $g_{d}$ is fermionic impurity coupling constant and $\mathbf{x}_{i}$ are the static positions of the quenched disorder. So the correlation function will turnout as $\left\langle V_{d}(-q) V_{d}(q)\right\rangle=\beta \delta_{i \omega_{m}, 0} \kappa$, where $q=\left(\mathbf{q}, i \omega_{m}\right)$. $\beta$ is known as the inverse temperature, where as $\omega_{m}=2 \pi m / \beta$ is the bosonic Matsubara frequency with $m$ being an integer. The disorder strength can be written as $\kappa=n_{i} g_{d}^{2}$, where $n_{i}$ being the concentration of the impurities. 
The partition function corresponding to the Hamiltonian can be written in the path integral formulation as

$$
Z=\int \mathcal{D}[\bar{\Psi}, \Psi] \exp [-S(\{\bar{\Psi}\}\{\Psi\})]
$$

where the action $S=\int_{0}^{\beta} d \tau \int d \mathbf{x}\left[\bar{\Psi}_{\sigma} \partial_{\tau} \Psi_{\sigma}+H\right]$. An introduction of pairing field $\Delta(\mathbf{x}, \tau)$ and applying the Grassman identity $\left(\int \mathcal{D}[\bar{\Delta}, \Delta] \exp \left[-\int d \mathbf{x} \int_{0}^{\beta} d \tau \bar{\Delta} \Delta / g\right]=\right.$ 1) in Eq.(1) the new partition function can be written as,

$$
Z=\int \mathcal{D}[\bar{\Psi}, \Psi] \int \mathcal{D}[\bar{\Delta}, \Delta] \exp \left[-S_{\text {eff }}\right]
$$

The effective action, $S_{\text {eff }}=S(\bar{\Psi}, \Psi)+1 / g \int d \mathbf{x} \int_{0}^{\beta} d \tau \bar{\Delta} \Delta$. Following HubbardStratonovich transformation, Eq.(2) can be written in terms of inverse Nambu propagator as,

$$
Z_{e f f}=\int \mathcal{D}[\bar{\Delta}, \Delta] e^{-1 / g \int d \mathbf{x} \int_{0}^{\beta} d \tau \bar{\Delta} \Delta} \times \int \mathcal{D}[\bar{\Psi} \Psi] e^{-\int d \mathbf{x} \int_{0}^{\beta} d \tau \bar{\Psi} G^{-1} \Psi},
$$

where the inverse Nambu propagator $G^{-1}(\mathbf{x}, \tau)$ is defined as, $G^{-1}=-\partial_{\tau} \mathbb{I}+$ $\left(\nabla^{2} / 2 m+\mu-V_{d}\right) \sigma_{z}+\Delta \sigma_{x}$ where $\mathbb{I}$ the identity matrix and $\sigma_{i}$ are the Pauli matrices $(i \in\{x, y, z\})$. So after integrating out the fermionic fields from Eq.(3) we are left with the effective action as,

$$
S_{\text {eff }}=\int d \mathbf{x} \int_{0}^{\beta} d \tau\left[\frac{|\Delta(\mathbf{r})|}{g}-\frac{1}{\beta} \operatorname{Tr} \ln \left\{-\beta G^{-1}(\mathbf{r})\right\}\right],
$$

where $\mathbf{r}=(\mathbf{x}, \tau)$. It is important to mention that the main contribution in the partition function comes from a small fluctuation $\delta \Delta(\mathbf{x}, \tau)=\Delta(\mathbf{x}, \tau)-\Delta$ where $\Delta$ is the homogeneous BCS pairing field. The original Green's function can be written as a sum of Green's function in absence of disorder $\left(G_{0}^{-1}=-\partial_{\tau} \mathbb{I}+\left(\nabla^{2} / 2 m+\mu\right) \sigma_{z}+\right.$ $\left.\Delta \sigma_{x}\right)$ and a self energy contribution $\left(\Sigma=-V_{d} \sigma_{z}+\delta \Delta \sigma_{+}+\delta \bar{\Delta} \sigma_{-}\right)$which contains the disorder as well as the small fluctuations of the BCS pairing fields, where $\sigma_{ \pm}$ are the ladder matrices.

It is possible to write the effective action $\left(S_{\text {eff }}\right)$ in Eq.(4) as a sum of bosonic action $\left(S_{B}\right)$ and fermionic action $\left(S_{F}\right)$ by expanding the inverse Nambu propagator upto the second order. In other words, it suggests that the expansion of the self energy is carried out till the first order in the disorder strength $(\kappa)$, which holds good as long as the disorder is weak. The effective action also contains a term, which emerges from the linear order of self energy expansion $\left(G_{0} \Sigma\right)$. It is possible to set the linear order to zero if we consider $S_{F}$ is an extremum of $S_{\text {eff }}$ after performing all the fermionic Matsubara frequency sums. The constrained condition leads to the BCS gap equation which after appropriate regularization through s-wave scattering 
length reads,

$$
-\frac{m}{4 \pi a}=\sum_{k}\left[\frac{1}{2 E_{k}}-\frac{1}{2 \epsilon_{k}}\right]
$$

This suggests that the BCS gap equation does not have any contribution from the disorder potential. These conditions holds well when one considers the disorder as weak. Now to construct the density equation with usual prescription of statistical mechanics; the thermodynamic potential $\Omega$ should be differentiated with respect to the chemical potential $\mu$. $\Omega$ can be written as a sum over fermionic $\left(\Omega_{F}\right)$ and bosonic $\left(\Omega_{B}\right)$ thermodynamic potentials. So density, $n=n_{F}+n_{B}=-\frac{\partial}{\partial \mu}\left(\Omega_{F}+\Omega_{B}\right)=$ $-\frac{1}{\beta} \frac{\partial}{\partial \mu}\left(S_{F}+S_{B}\right)$. The well known BCS density equation can be restored if we consider only $n_{F}$ but the presence of disorder and fluctuation leads to $n_{B} \neq 0$. Hence the final mean field density equation will be,

$$
n=\sum_{k}\left(1-\frac{\xi_{k}}{E_{k}}\right)-\frac{\partial \Omega_{B}}{\partial \mu},
$$

where $\Omega_{B}$ is defined as,

$$
\Omega_{B}=\lim _{\beta \rightarrow \infty} \frac{1}{2 \beta} \sum_{q} \ln \operatorname{det} M-\frac{\kappa}{2} \sum_{\mathbf{q}, \omega_{m}=0} W^{\dagger} M^{-1} W .
$$

The first term in Eq.(7) is the contribution arises due to the fluctuating pairing fields. This contribution becomes important if the objective of study relates to the finite temperature effect on the system. Since our purpose of study is the effect of disorder at zero temperature, from here on we will neglect this contribution. In Eq.(7), $W$ is a doublet which couples the disorder with the fluctuation. At $T=0$ after performing the fermionic Matsubara frequency summation one finds,

$$
W_{1}=W_{2}=\sum_{k} \frac{\Delta\left(\xi_{k}+\xi_{k+q}\right)}{2 E_{k} E_{k+q}\left(E_{k}+E_{k+q}\right)} .
$$

The inverse fluctuation propagator is a $2 \times 2$ symmetric matrix and at zero temperature it reads,

$$
\begin{aligned}
& M_{11}=\frac{1}{g}+\sum_{k}\left[\frac{v_{k}^{2} v_{k+q}^{2}}{i \omega_{m}-E_{k}-E_{k+q}}-\frac{u_{k}^{2} u_{k+q}}{i \omega_{m}+E_{k}+E_{k+q}}\right] \\
& M_{12}=\sum_{k} u_{k} v_{k} u_{k+q} v_{k+q}\left[\frac{1}{i \omega_{m}+E_{k}+E_{k+q}}-\frac{1}{i \omega_{m}-E_{k}-E_{k+q}}\right],
\end{aligned}
$$

with $M_{22}(q)=M_{11}(-q)$ and $M_{21}(q)=M_{12}(q)$. The other functions bear usual BCS notation with $\xi_{k}=\mathbf{k}^{2} / 2 m-\mu, E_{k}=\sqrt{\xi_{k}^{2}+\Delta^{2}}, u_{k}^{2}=1 / 2\left(1+\xi_{k} / E_{k}\right)$ and $v_{k}^{2}=1 / 2\left(1-\xi_{k} / E_{k}\right)$. 

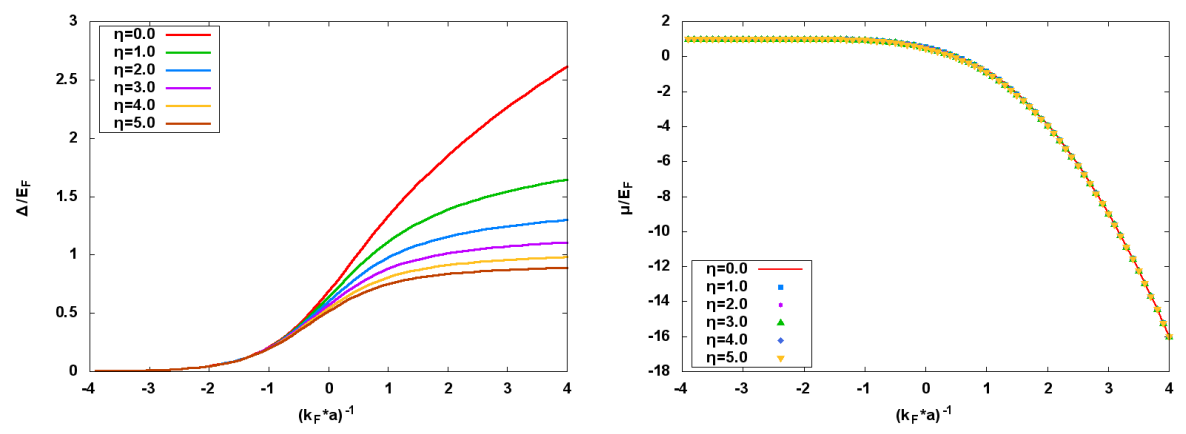

Fig. 1. The order parameter follows BCS mean field approximation of exponential decay in the BCS side, whereas in the BEC side it is more prone to the disorder and gets depleted due to destruction of superfluidity. Conversely the chemical potential remains unaffected.

Finally the disorder induced bosonic thermodynamic potential at zero temperature reads:

$$
\Omega_{B}=\frac{\kappa}{2} \sum_{\mathbf{q}} \frac{2 W_{1}^{2}}{M_{11}+M_{12}}
$$

where the elements of the pair fluctuation matrix takes the form,

$$
M_{11}+M_{12}==\sum_{k}\left[\frac{\Delta^{2}-E_{k} \xi_{k+q}-E_{k+q} \xi_{k}}{2 E_{k} E_{k+q}\left(E_{k}+E_{k+q}\right)}+\frac{1}{2 E_{k}}\right] \text {. }
$$

\section{Result and Discussion}

Eq.(5) and (6) are now ready to be solved self consistently, aided by the results of Eq.(8-11). It is clear from these equations that the disorder strength $\kappa$ is an input parameter, this provokes the question about how to estimate the disorder strength as weak. Firstly $\kappa$ has a dimension of $k_{F} / m^{2}$ which leads to the dimensionless disorder strength $\eta=\kappa m^{2} / k_{F}$. But a more physical description can be worked out if the impurity strength is normalized by Fermi density and square of Fermi energy $^{18}$. According to the this new definition, $\tilde{\eta}=\kappa n_{F} / \epsilon_{F}^{2}=4 /\left(3 \pi^{2}\right) \eta$. Therefore it is essential that the impurity density and strength remain much less than Fermi density and Fermi energy.

Fig.1 demonstrates that in the BCS limit the bosonic contribution becomes nominal. As a result the order parameter with different disorder strength follows the mean field approximation of $\Delta / \epsilon_{F}=8 e^{-2} \exp \left[-\pi /\left(2 k_{F} a\right)\right]$ corresponding to $1 / k_{F} a \rightarrow-\infty$ thus emphasizing the validity of Anderson theorem ${ }^{4}$. In the BEC limit it is possible to make analytic extension for the bosonic thermodynamic potential by expanding the inverse fluctuation propagator matrix elements in powers of $q^{20}$. In effect the coefficients of $q$ turns up simple analytic integrals. A systematic and careful calculation leads to an analytic description of the order parameter in this 
limit, where one can observe a progressive depletion. Here the impurity actually starts to destroy the superfluidity and in effect the order parameter is depleted and the fraction of depletion remains in the order of $\eta /\left(k_{F} a\right)$ in agreement with Ref.[15]. Hence Anderson theorem breaks down in this limit. The chemical potential, expectantly remains pinned to the Fermi energy in the BCS limit and follows the ideal path (without disorder situation) towards the BEC limit.

The present study explicitly reveals that the disorder starts to play its role in the crossover window (which is usually considered as $1 / k_{F} a \simeq[-1,1]$ ) itself. It suggests, that the depletion of order parameter in vicinity of unitarity leads to the onset of superfluid destruction. Though there exist numerous discussions on the BCS and BEC extremes with disorder, but according to best of our knowledge, the behavior of the disorder induced order parameter is not yet been reported. Here for the first time we show this behavior from weak to strong coupling limit.

\section{Conclusion}

In conclusion, we included an impurity like weak disorder through the gaussian fluctuation route as prescribed earlier ${ }^{15,18}$ and then solved the coupled BCS mean field equation self consistently. This enabled us to obtain the two mean field parameters $\Delta$ and $\mu$ where we show that the order parameter gets depleted leaving the chemical potential unchanged. These behaviors were commented in Ref.[15] but here we present the exact calculation for the first time. The gradual decrease of $\Delta$ can be connected to the destruction of superfluidity. It is already known ${ }^{13}$ that the random potential destroys the superfluid nature in Bose gas hence the superfluid order parameter gets depleted. Further it reveals that the destructive process begins around the resonance point. We are now in process in understanding other aspects using this formalism ${ }^{21}$. In future it will be very interesting to study the crossover region under the influence of large impurity to fill the void of our knowledge in this situation. Therefore we hope a very exciting future for this fairly new topic in the coming days.

\section{Acknowledgments}

A.K would like to thank the Post Doctoral Grant from Research Center for Dielectric and Advanced Matter Physics, Pusan National University.

\section{References}

1. C. Chin, R. Grimm, P. Julienne, and E. Tiesinga, Rev. Mod. Phys. 82, 1225 (2010).

2. S. Georgini, L. P. Pitaevskii, and S. Stringari, Rev. Mod. Phys. 80, 1215 (2008).

3. I. Bloch, J. Dalibard, and W. Zwerger, Rev. Mod. Phys. 80, 885 (2008).

4. P. W. Anderson, J. Phys. Chem. Solids 11, 26 (1959).

5. J. Billy et. al., Nature 453, 891 (2008).

6. G. Roati et. al., Nature 453, 895 (2008).

7. S. S. Kondov, W. R. McGhee, J. J. Zirbel, and B. DeMarco, Science 334, 66 (2011). 
8. F. Jendrzejewski et. al., arXiv:1108.013\%.

9. L. S. Palencia, and M. Lewenstein, Nature Phys. 6, 87 (2010).

10. D. Belitz, and T. R. Kirkpatrick, Rev. Mod. Phys. 66, 261 (1994).

11. A. Ghoshal, M. Randeria and N. Trivedi, Phys. Rev. B 65, 014501 (2001).

12. K. Bouadim, Y. L. Loh, M. Randeria, and Nandini Trivedi, Nature Phys. 7, 884 (2011).

13. K. Huang, and H. F. Meng, Phys. Rev. Lett. 69, 644 (1992).

14. S. Giorgini, L. Pitaevskii, and S. Stringari, Phys. Rev. B 49, 12938 (1994).

15. G. Orso, Phys. Rev. Lett. 99, 250402 (2007).

16. B. I. Shklovskii, Superconductors 42, 909 (2008).

17. C. A. R. Sá de Melo, Physics Today 61, 45 (2008).

18. L. Han, and C. A. R. Sá de Melo, New J. Phys. 13, 055012 (2011).

19. P. Nozières S. Smith-Rink, J. Low Temp. Phys. 59, 195 (1985).

20. E. Taylor, A. Griffin, N. Fukushima, and Y. Ohashi, Phys. Rev. A 74, 063626 (2006).

21. A. Khan, S. Basu and S. W. Kim, arXiv:1202.5117. 